\title{
The postprandial state does not impair endothelial function in women with Type 2 diabetes irrespective of glycaemic control
}

\author{
C. H. Strey ${ }^{1}$ J. Young ${ }^{1}$ M. Mollier ${ }^{1}$ C. M. Florkowski ${ }^{1}$ B. I. Shand ${ }^{1}$ - R. S. Scott ${ }^{1}$ \\ ${ }^{1}$ Lipid and Diabetes Research Group, Hagley Building, Christchurch Hospital, Christchurch, New Zealand
}

\begin{abstract}
Aims/hypothesis. The postprandial state has been shown to be associated with endothelial dysfunction, a predictor of cardiovascular morbidity. In Type 2 diabetes, postprandial metabolic excursions are prolonged and exaggerated, but less pronounced if glycaemic control is optimised. We investigated the impact of improved glycaemic control on endothelial function in the postprandial state.

Methods. We studied 19 postmenopausal women with Type 2 diabetes and ten non-diabetic subjects. Participants with diabetes were re-studied 3 months after intensive glucose regulation. We measured forearm

following an $\mathrm{HbA}_{1} \mathrm{c}$ reduction of $0.96 \%$ ( $p<0.05$ for high-dose acetylcholine infusion). Postprandial metabolic excursions were higher in the diabetic group $(p<0.001, p<0.01$ and $p<0.05$ for glucose, insulin and triglycerides respectively). Resting forearm blood flow increased in all groups after the meal $(p<0.005)$. There was no difference in fasting and postprandial endothelium-dependent vasodilation before and after improved glucose regulation in either group.

Conclusions/interpretation. The postprandial state does not impair endothelial function in non-diabetic women and does not make pre-existing endothelial dysfunction worse in women with Type 2 diabetes, irrespective of glycaemic control.
\end{abstract} blood flow by strain gauge plethysmography during rest, during acetylcholine infusion and post ischaemia in the fasting state, and again 3 hours after a mixed meal (660 kcal, 55\% fat).

Results. Endothelium-dependent vasodilation was impaired in the diabetic group $(p<0.005)$ and improved

Keywords Endothelial function .

Forearm plethysmography · Glycaemic control · Postprandial state . Type 2 diabetes
Received: 15 March 2004 / Accepted: 10 June 2004

Published online: 22 October 2004

(C) Springer-Verlag 2004

\section{R. S. Scott (}

Lipid and Diabetes Research Group, Hagley Building, Christchurch Hospital, 8001 Christchurch, New Zealand

E-mail: russell.scott@chmeds.ac.nz

Tel.: +64-3-3641091, Fax: +64-3-3640457

Abbreviations: EDV, endothelial-dependent vasodilation . $\mathrm{FBF}$, forearm blood flow $\cdot \triangle \mathrm{FBF}$, increase above resting forearm blood flow $\cdot \mathrm{rFBF}$, ratio of forearm blood flow in the infused arm to forearm blood flow in the control arm - FMD, flow-mediated dilation - MAP, mean arterial blood pressure

\section{Introduction}

Postprandial metabolic excursions are exaggerated in people with insulin resistance [1]. In Type 2 diabetes postprandial hyperglycaemia is an independent risk factor for cardiovascular disease [2] and a stronger predictor of cardiovascular mortality than fasting plasma glucose [3]. There is mounting evidence to suggest that delayed postprandial lipaemia represents an independent cardiovascular risk factor [4]. Endothelial dysfunction precedes [5] and predicts [6] overt cardiovascular disease and is therefore a potential mechanism linking the postprandial state to cardiovascular risk [7]. The extent of endothelial impairment can be assessed clinically by strain gauge plethysmography 
[8]. In this study we assessed how the ingestion of a fatty meal affects endothelium-dependent vasodilation of forearm resistance vessels in non-diabetic women and in women with Type 2 diabetes before and after improvement of glycaemic control. We hypothesised that a test meal would impair endothelial function more in participants with Type 2 diabetes than in nondiabetic subjects, as postprandial metabolic excursions are exaggerated and prolonged in Type 2 diabetes [9]. Consequently, reduced metabolic excursions, achieved through better diabetic control, should result in less postprandial impairment of endothelial function.

\section{Subjects and methods}

Subjects. Nineteen women aged between 50 and 77 years with established Type 2 diabetes (according to the WHO criteria for the diagnosis of diabetes) were recruited from the Diabetes Service at Christchurch Hospital, New Zealand. For inclusion in the study, an $\mathrm{HbA}_{1} \mathrm{c}$ level of above $7.6 \%$ was required. In order to minimise patient variability, only postmenopausal women who were not on hormone replacement therapy were recruited. The postmenopausal state was defined as the absence of menstruation for more than 2 years and a folliclestimulating hormone level of above $25 \mathrm{U} / 1$. The main exclusion criteria included a history of advanced renal failure, unstable angina, atrial fibrillation, serious allergic reactions, clotting disorders and use of warfarin, oestrogen and cortisol-containing preparations. For the non-diabetic group we recruited ten previously healthy, postmenopausal women through advertisements in local newspapers. Participants in the non-diabetic group were on no medications. Written informed consent was obtained from all subjects. The Canterbury Ethics Committee approved the study protocol.

Study protocol. At the screening visit, medical history, physical examination with anthropoid measurements, deep breathing test and blood sample collections (plasma glucose, electrolytes, renal and liver tests, haematology and $\mathrm{HbA}_{1} \mathrm{c}$ ) were performed. The first study visit followed 2 to 4 days later. Participants attended during fasting, having not smoked for 12 hours and having refrained from morning exercise. Study visits took place in the Medical Research Centre of the Department of Medicine, Christchurch School of Medicine, a quiet, windowless and air-conditioned room (constant temperature $22^{\circ} \mathrm{C}$ ). The study protocol was the same for all visits. At 07.30 hours, the participants lay down in a supine position for at least $15 \mathrm{~min}$. Fasting venous blood samples were then collected for glucose, $\mathrm{HbA}_{1} \mathrm{c}$, insulin, cardiovascular risk markers, lipoproteins and endothelin-1, and were processed immediately. A 22g plastic cannula (Radial Artery Catheterization Set, Arrows International, Germany) was inserted into the brachial artery of the non-dominant arm near the cubital fossa under local anaesthesia, and forearm blood flow was assessed by venous occlusion plethysmography. Blood pressure was measured in triplicates, in the dominant (non-infused) arm using a standard sphygmometer, by the same investigator before, during and after blood flow studies with the participant remaining supine. Following the first plethysmography session, participants were guided to a comfortable armchair in an adjacent observation room. The arterial needle remained in situ with the extended arm immobilised by a splint. A 660-kcal breakfast (croissants, ham, cheese, polyunsaturated margarine, bread, tomatoes, eggs and water) was served at 10.30 hours, with $57 \%$ of energy pro- vided from fat (41 g), 26\% provided from carbohydrate (45 g) and $17 \%$ provided from protein $(26 \mathrm{~g})$. Participants remained seated for 3 hours after the meal. Postprandial blood tests and blood flow studies were repeated in an identical fashion as in the fasting state. The non-diabetic participants were discharged at the end of the first study visit. Participants with Type 2 diabetes entered a three-month interventional phase commencing at the first study visit. All subjects received individualised education and advice from a dietician and a physician. During the advice session with the physician, exercise strategies were outlined and initial medication adjustments were made, adding and/or increasing oral hypoglycaemic therapy and/or insulin therapy. Participants were encouraged to check capillary blood glucose levels three to six times daily and to keep a blood glucose diary. The physician contacted each participant at least every fortnight to further optimise therapy. Treatment targets were fasting capillary blood glucose levels of between 4 and $7 \mathrm{mmol} / \mathrm{l}$ and 2 -h postprandial levels of less than $10 \mathrm{mmol} / \mathrm{l}$. Participants were commenced on insulin if treatment targets could not be reached despite maximal metformin and sulphonylurea therapy. Three participants were changed from a twicedaily to a four-times-daily basal-bolus insulin regimen. All participants were invited to see the dietician a second time during the second month of the intervention. Post intervention, fasting and postprandial blood tests as well as blood flow studies were repeated in an identical fashion.

Venous occlusion plethysmography. The participant lay with both forearms placed in braces above the level of the heart. Appropriately sized mercury-filled silastic strain gauges (Hokanson, Bellevue, Wash., USA) were placed around the widest section of each forearm and connected to a plethysmograph (EC-4; Hokanson), which was interfaced with a computer. After infusing physiological saline for $30 \mathrm{~min}$ at a rate of $1 \mathrm{ml} / \mathrm{min}$ (Terumo constant rate infuser) through the arterial line, wrist cuffs were inflated to $50 \mathrm{~mm} \mathrm{Hg}$ above arterial pressure before the measurements were taken. Forearm blood flow of the non-dominant (infused) forearm was measured in the following sequences: (i) resting; (ii) during intra-arterial infusion of acetylcholine at 20 and $40 \mu \mathrm{g} / \mathrm{min}$ (low- and highdose); (iii) resting after a break of $10 \mathrm{~min}$; and (iv) after local ischaemia induced by brachial cuff inflation to $50 \mathrm{~mm} \mathrm{Hg}$ above arterial pressure for $5 \mathrm{~min}$. Resting blood flow of the dominant (non-infused) arm was measured simultaneously. Using self-developed software, forearm blood flow was derived from forearm distension and expressed as millilitres per $100 \mathrm{ml}$ forearm tissue per minute $\left(\mathrm{ml} \cdot \mathrm{min}^{-1} \cdot \mathrm{dl}^{-1}\right)$. Forearm distension was achieved by inflating and deflating brachial cuffs to supra-venous pressure levels $(45 \mathrm{~mm} \mathrm{Hg})$ with a rapid cuff inflator (E20; Hokanson) for 5 and 10 seconds respectively. Ten inflation-deflation cycles were recorded for each stage of the protocol and averaged. Our between-week CV for blood flow studies was $13 \%$ resting and $14 \%$ post ischaemia.

Biochemical tests. Lipid ultracentrifugation was performed in a Beckman L8-M Ultracentrifuge fitted with a Kontron TFT 45.6 Rotor at 37,000 rpm for 5 min using various density solutions. Lipoprotein fractions were separated using a tube slicer and the lipoprotein content of each fraction was measured after precipitation with phosphotungstate (Cobas BIO HDL method). Insulin was measured using a Roche Elecsys 2010 automated analyser after pre-treatment of the specimen with polyethylene glycol. Endothelin-1 was assayed using endothelin antiserum (catalogue number RAS6091; Peninsula Labs, Belmont, Calif., USA). High-sensitivity C-reactive protein was measured by particle-enhanced immunonephelometry on the Dade Behring BN2 Nephelometer (Dade Behring Marburg, 
Table 1. Characteristics of study participants

\begin{tabular}{|c|c|c|}
\hline & Non-diabetic group $(n=10)$ & Diabetic group $(n=19)$ \\
\hline Age (years) & $58.0 \pm 6.9$ & $60.6 \pm 7.1$ \\
\hline Smokers & $1 / 9(11 \%)$ & $3 / 16(19 \%)$ \\
\hline Mean arterial pressure (mm Hg) & $92 \pm 10$ & $105 \pm 17 *$ \\
\hline BMI $\left(\mathrm{kg} / \mathrm{m}^{2}\right)$ & $28.1 \pm 7.5$ & $36.1 \pm 6.1 * *$ \\
\hline Body fat $(\%)$ & $41 \pm 16$ & $54 \pm 9 *$ \\
\hline On sulphonylurea, $n$ ( $n$ commenced) & - & $8(2)$ \\
\hline On insulin, $n$ ( $n$ commenced) & - & $12(4)$ \\
\hline Insulin dose at baseline (units) & - & $71.1 \pm 19.6$ \\
\hline Insulin dose at end of study (units) & - & $74.5 \pm 27.9^{a}$ \\
\hline
\end{tabular}

Values are means \pm SD. E/I ratio, expiration : inspiration ratio. a $86.8 \pm 21.0$ units/day excluding those commenced on insulin during the study;

$* p<0.05 ; * * p<0.01 ; * * * p<0.001$

Germany). For the deep breathing test, a measure of parasympathetic heart rate control, a resting electrocardiogram was recorded continuously during four cycles of deep inspiration-expiration. The ratios of the longest to the shortest RR intervals for each respiratory cycle were averaged.

Data analysis. Changes in resting forearm blood flow (FBF) during acetylcholine infusions and post ischaemia were either expressed as the increase above resting blood flow $(\triangle \mathrm{FBF})$ to correct for meal-induced FBF changes, or as the ratio of blood flow in the infused arm to blood flow in the non-infused arm (ratio, $r \mathrm{FBF}$ ) to correct for systemic FBF changes [10]. $\Delta \mathrm{FBF}$ and $r \mathrm{FBF}$ were analysed by repeated measures ANOVA (general linear model). Paired and unpaired two-tailed $t$ tests, or Mann-Whitney $U$ test and Wilcoxon signed ranks test were used for comparison of variables between the diabetic and nondiabetic groups as well as within the diabetic group. Correlation analysis was performed using Pearson's or Spearman's correlation coefficient. Statistical significance was accepted at the $95 \%$ confidence interval $(p<0.05)$.

\section{Results}

Characteristics of study participants. The characteristics of the participants in the non-diabetic and diabetic groups are shown in Table 1. The insulin dose was increased by $19.0 \pm 17.4$ units in seven participants and decreased by 8 units in one participant, who was changed to a basal-bolus regimen. The average insulin dose in the four participants commenced on insulin was $50 \pm 24.0 \mathrm{pmol} / \mathrm{l}$. Plasma oestradiol was closely correlated with BMI $(r=0.633, p<0.0001)$. The expiration : inspiration ratio was significantly reduced among participants in the diabetic group.

Biochemical data. Fasting and postprandial blood results for the non-diabetic and diabetic groups before and after intervention are summarised in Table 2. In the fasting state, glycaemic indices differed significantly between the groups as expected. VLDL cholesterol, triglycerides and VLDL triglycerides were high- er, and HDL was lower in the diabetic group. The difference in biochemical cardiovascular risk markers, although higher in the diabetic group, did not reach statistical significance ( $p=0.22$ for high-sensitivity C-reactive protein, $p=0.13$ for fibrinogen and $p=0.082$ for endothelin-1). In the postprandial state, plasma insulin, VLDL cholesterol, triglycerides and VLDL triglycerides were increased compared with in the fasting state in both the non-diabetic and diabetic groups. Plasma glucose increased only in the diabetic group. Plasma glucose, plasma insulin, VLDL cholesterol, triglycerides and VLDL triglycerides were higher, and HDL was lower, in the diabetic group compared with in the non-diabetic group. Intensive glycaemic control for 3 months led to a reduction in $\mathrm{HbA}_{1} \mathrm{c}$ of $0.96 \%$. Fasting and postprandial plasma glucose was lowered without a change in fasting or postprandial insulin $(p=0.77, p=0.81)$. The positive effect on lipoprotein fractions, both fasting and postprandial, did not reach significance levels (increase in fasting HDL $p=0.063$, decrease in fasting and postprandial triglycerides $p=0.12$ and $p=0.16$ respectively, decrease in fasting and postprandial VLDL triglycerides $p=0.11$ and $p=0.27$ respectively).

Blood flow studies. Table 3 summarises FBF, $\triangle \mathrm{FBF}$ and $r$ FBF data. Figure 1 shows the effect of acetylcholine infusion on fasting and postprandial FBF, and Figure 2 shows the effect of the meal on $\triangle F B F$ in both groups before and after intervention.

In the fasting state, resting FBF did not differ between the groups $(p=0.25)$. However, $\triangle \mathrm{FBF}$ during high- and low-dose acetylcholine infusion and post ischaemia was reduced in the diabetic group. This between-group difference was more significant when expressed as $r$ FBF.

In the postprandial state, resting FBF was increased in both groups. In the fasting state, resting FBF did not differ between the groups $(p=0.34)$, but $\Delta \mathrm{FBF}$ and 
Table 2. Fasting and postprandial biochemical data in the non-diabetic and diabetic groups before and after intervention

Non-diabetic group Diabetic group pre intervention Diabetic group post intervention

Fasting

$\mathrm{HbA}_{1} \mathrm{c}(\%)$

Plasma glucose $(\mathrm{mmol} / \mathrm{l})$

Plasma insulin ( $\mathrm{pmol} / \mathrm{l})$

LDL cholesterol (mmol/l)

HDL cholesterol (mmol/l)

Triglycerides ( $\mathrm{mmol} / \mathrm{l})$

VLDL cholesterol (mmol/l)

VLDL triglycerides (mmol/l)

UACR (g/mol)

Endothelin-1 (pg/ml)

$\mathrm{HsCRP}(\mathrm{mg} / \mathrm{l})$

Fibrinogen (g/l)

Postprandial

Plasma glucose (mmol/l)

Plasma insulin (pmol/l)

LDL cholesterol (mmol/l)

HDL cholesterol (mmol/l)

Triglycerides ( $\mathrm{mmol} / \mathrm{l})$

VLDL cholesterol (mmol/l)

VLDL triglycerides ( $\mathrm{mmol} / \mathrm{l})$

$$
\begin{gathered}
5.68 \pm 0.16 \\
4.78 \pm 0.15 \\
67.0 \pm 16.5 \\
3.2 \pm 0.28 \\
1.46 \pm 0.13 \\
1.10 \pm 0.17 \\
0.37 \pm 0.07 \\
0.56 \pm 0.13 \\
0.54 \pm 0.06 \\
1.22 \pm 0.11 \\
2.57 \pm 0.98 \\
2.97 \pm 0.24
\end{gathered}
$$

$4.6 \pm 0.17$
$162.2 \pm 50.3^{\#}$
$3.15 \pm 0.22$
$1.40 \pm 0.14$
$1.70 \pm 0.27^{\#}$
$0.52 \pm 0.09^{\#}$
$1.00 \pm 0.21^{\#}$

$8.67 \pm 0.25 * * *$
$11.61 \pm 0.95 * * *$
$144.1 \pm 22.3 * *$
$2.79 \pm 0.14$
$1.07 \pm 0.08^{* *}$
$1.95 \pm 0.24 *$
$0.65 \pm 0.11$
$1.13 \pm 0.19 *$
$16.56 \pm 7.84 *$
$1.53 \pm 0.137$
$5.82 \pm 1.55$
$3.39 \pm 0.15$

$15.64 \pm 1.35 * * * \# \#$

$292.2 \pm 41.9 * * \#$

$2.67 \pm 0.14$

$1.03 \pm 0.07 *$

$2.92 \pm 0.37 * \#$

$0.87 \pm 0.14^{\# \#}$

$1.91 \pm 0.32^{\# \#}$
$7.71 \pm 0.30 \S$
$8.92 \pm 1.248$
$140.8 \pm 23.2$
$2.82 \pm 0.12$
$1.19 \pm 0.10^{\S}$
$1.77 \pm 0.18$
$0.58 \pm 0.08$
$0.96 \pm 0.15$
$15.00 \pm 8.94$
$0.66 \pm 0.13$
$6.85 \pm 1.79$
$3.41 \pm 0.23$

$12.89 \pm 1.34^{\# \# \S ~}$

$300.7 \pm 44.3^{\# \#}$

$2.57 \pm 0.16$

\begin{tabular}{|c|c|c|c|c|c|c|}
\hline & \multicolumn{3}{|l|}{ Fasting } & \multicolumn{3}{|l|}{ Postprandial } \\
\hline & $\begin{array}{l}\text { Non-diabetic } \\
\text { group }\end{array}$ & $\begin{array}{l}\text { Diabetic } \\
\text { group pre } \\
\text { intervention }\end{array}$ & $\begin{array}{l}\text { Diabetic } \\
\text { group post } \\
\text { intervention }\end{array}$ & $\begin{array}{l}\text { Non-diabetic } \\
\text { group }\end{array}$ & $\begin{array}{l}\text { Diabetic } \\
\text { group pre } \\
\text { intervention }\end{array}$ & $\begin{array}{l}\text { Diabetic } \\
\text { group post } \\
\text { intervention }\end{array}$ \\
\hline $\begin{array}{l}\text { Mean arterial blood } \\
\text { pressure }(\mathrm{mm} \mathrm{Hg})\end{array}$ & $91.2 \pm 3.2$ & $104.5 \pm 4.0 *$ & $100.3 \pm 3.8$ & $89.2 \pm 2.8$ & $103.0 \pm 4.0 *$ & $99.6 \pm 3.6$ \\
\hline FBF resting & $2.75 \pm 0.37$ & $3.27 \pm 0.28$ & $2.77 \pm 0.21$ & $4.04 \pm 0.75^{\# \#}$ & $4.62 \pm 0.39 \# \#$ & $4.56 \pm 0.41^{\# \#}$ \\
\hline $\begin{array}{l}\Delta \text { FBF during low-dose } \\
\text { acetylcholine infusion }\end{array}$ & $14.68 \pm 2.12$ & $9.26 \pm 1.25^{*}$ & $10.66 \pm 1.42$ & $15.62 \pm 2.14$ & $10.29 \pm 1.53^{*}$ & $12.42 \pm 1.54$ \\
\hline $\begin{array}{l}\Delta \mathrm{FBF} \text { during high-dose } \\
\text { acetylcholine infusion }\end{array}$ & $18.69 \pm 2.61$ & $10.48 \pm 1.30 * *$ & $13.65 \pm 1.67 \S$ & $18.82 \pm 2.50$ & $11.74 \pm 1.52^{* *}$ & $13.90 \pm 1.52$ \\
\hline$\Delta \mathrm{FBF}$ post ischaemia & $24.76 \pm 3.54$ & $13.75 \pm 2.09 * *$ & $13.12 \pm 2.27$ & $23.47 \pm 3.10$ & $13.21 \pm 1.80 * *$ & $14.10 \pm 1.80$ \\
\hline $\begin{array}{l}r \text { FBF during low-dose } \\
\text { acetylcholine infusion }\end{array}$ & $7.8 \pm 1.1$ & $4.2 \pm 0.5^{* * *}$ & $5.2 \pm 0.6$ & $8.3 \pm 1.4$ & $4.4 \pm 0.5^{* *}$ & $5.3 \pm 0.5$ \\
\hline $\begin{array}{l}r \text { FBF during high-dose } \\
\text { acetylcholine infusion }\end{array}$ & $9.1 \pm 1.0$ & $4.4 \pm 0.4 * * *$ & $6.3 \pm 0.7 \S$ & $9.2 \pm 1.2$ & $4.9 \pm 0.5 * * *$ & $5.7 \pm 0.5$ \\
\hline$r \mathrm{FBF}$ post ischaemia & $12.3 \pm 2.1$ & $6.3 \pm 0.9 * *$ & $5.5 \pm 1.0$ & $12.0 \pm 1.8$ & $6.7 \pm 0.9 * *$ & $5.6 \pm 0.5$ \\
\hline
\end{tabular}

$1.04 \pm 0.08$

$2.54 \pm 0.28^{\# \#}$

$0.77 \pm 0.09 \#$

$1.66 \pm 0.23^{\# \#}$

Values are means \pm SEM. UACR, urinary albumin creatinine ratio; hsCRP, high-sensitivity $\mathrm{C}$-reactive protein.

$* p<0.05$, ** $p<0.01$, *** $p<0.001$ for differences between the

ferences between fasting and postprandial; $\S p<0.05, \S \S p<0.01$ diabetic and non-diabetic group; \# $p<0.05$, \#\# $p<0.001$ for dif-

Table 3. Mean arterial blood pressure and forearm blood flow in the non-diabetic and diabetic groups before and after intervention in the fasting and postprandial states

Values are means \pm SEM. $* p<0.05$, ** $p<0.01$, *** $p<0.001$ for differences between the diabetic and non-diabetic groups; $\# \# p<0.005$ for differences between fasting and postprandial; ${ }^{\S} p<0.05$ for differences between pre and post intervention

$r$ FBF were reduced in the diabetic group. There was no difference in $\triangle \mathrm{FBF}$ or $r \mathrm{FBF}$ in the postprandial state compared with in the fasting state in either group (for acetylcholine infusion $p=0.88$ and $p=0.77$ in the non-diabetic group, $p=0.29$ and $p=0.28$ in the diabetic group; for post ischaemia $p=0.15$ and $p=0.96$ in the non-dia- betic group, $p=0.79$ and $p=0.71$ in the diabetic group). After 3 months of intensive glycaemic control there was a trend for an increase in $\triangle \mathrm{FBF}$ and $r \mathrm{FBF}$ during acetylcholine infusion $(p=0.085$ and $p=0.056$ respectively), reaching statistical significance for high-dose acetylcholine infusion in the fasting state. Improve- 


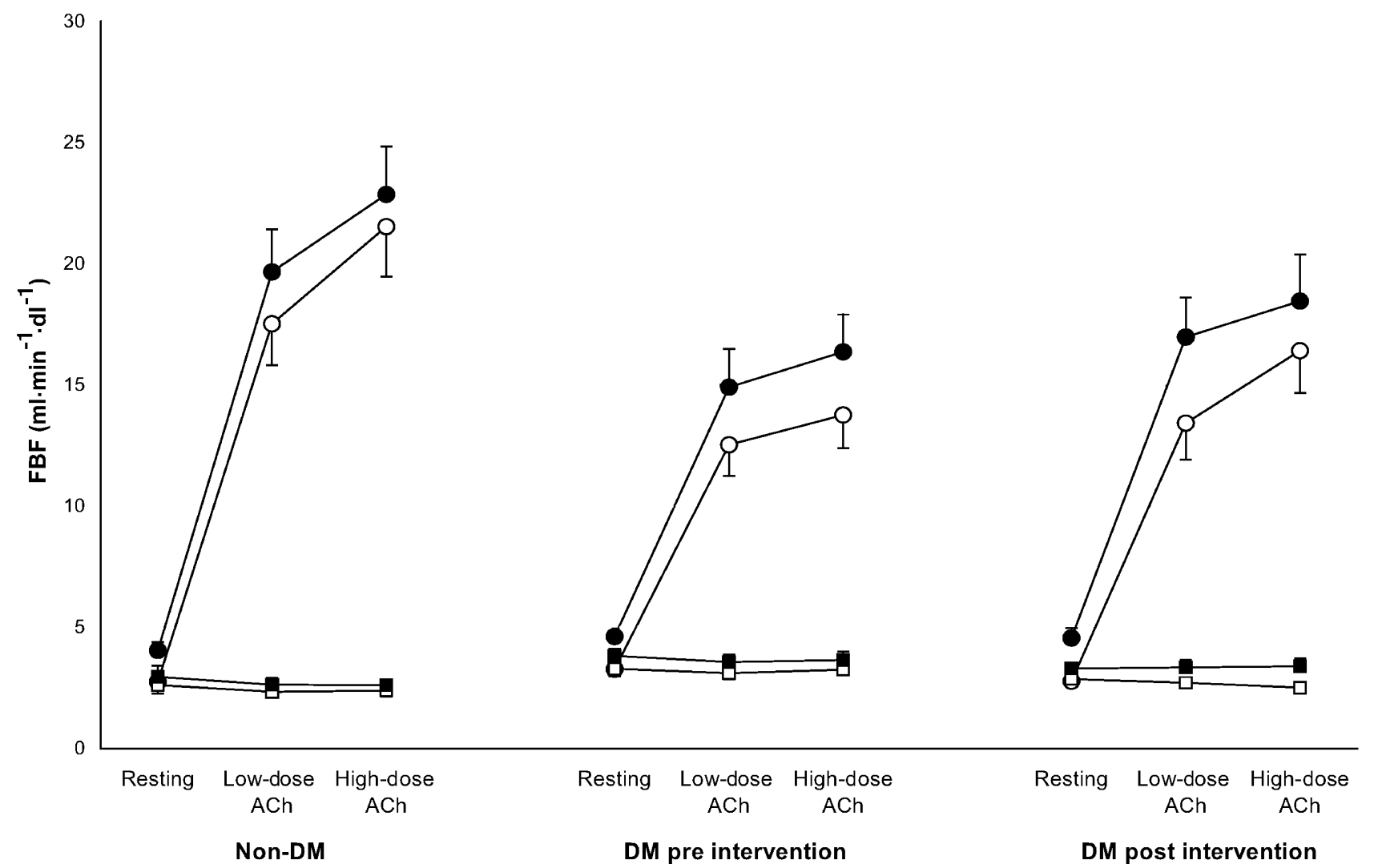

Fig. 1. Effect of intra-arterial acetylcholine (ACh) infusion on fasting (empty markers) and postprandial (full markers) FBF in the non-diabetic (Non-DM) and diabetic (DM) groups before and after

intervention. Squares, control arm; circles, infused arm. Data are presented as means \pm SEM. Please refer to Table 3 for the significance levels of the differences between groups and interventions

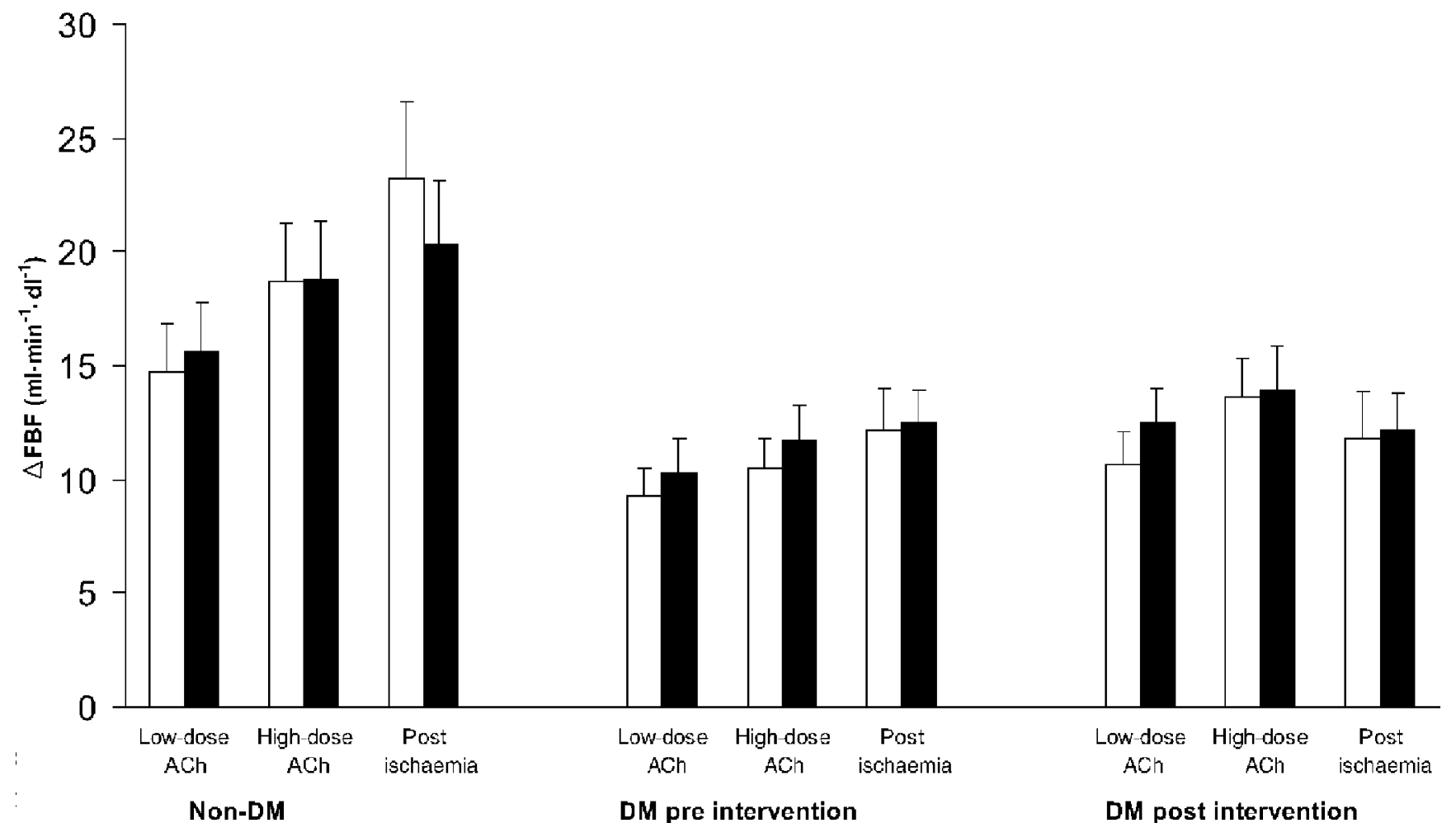

Fig. 2. Fasting (empty bars) and postprandial (full bars) $\triangle F B F$ in the non-diabetic (Non-DM) and diabetic (DM) groups before and after intervention. Data are presented as means \pm

SEM. ACh, acetylcholine. Please refer to Table 3 for significance levels of the differences between groups and of intervention-induced differences. 
Fig. 3. Correlation of plasma insulin and $\triangle \mathrm{FBF}$ during high-dose acetylcholine infusion in the non-diabetic group (squares) and the diabetic group (circles) in the fasting state (empty markers) and the postprandial state (full markers). Plasma insulin is presented on a logarithmic scale to account for its abnormal distribution

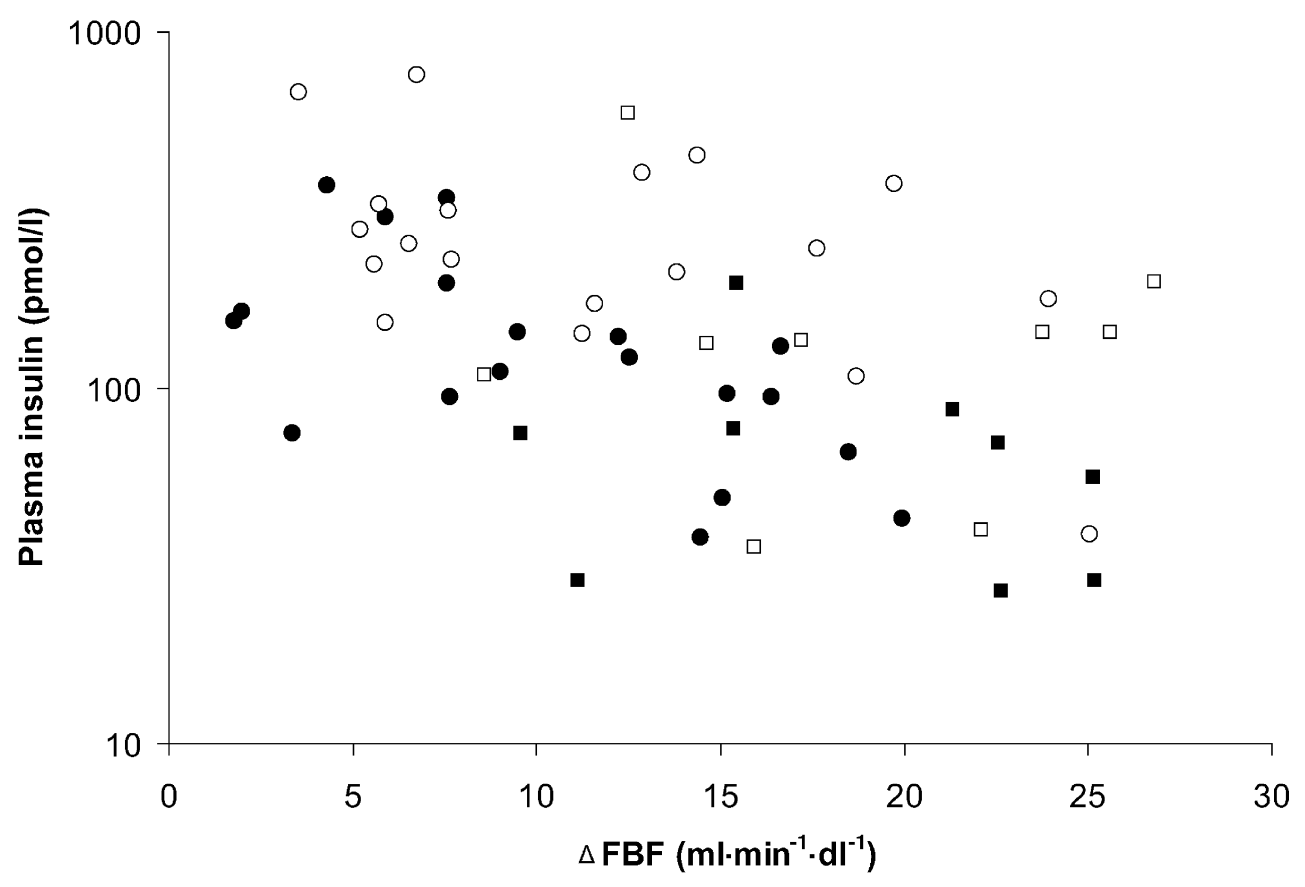

ments in glycaemic control had no significant effect on $\Delta \mathrm{FBF}$ and $r \mathrm{FBF}$ post ischaemia (fasting $p=0.96$ and $p=0.97$, postprandial $p=0.37$ and $p=0.95$ ). Mirroring results before intensive glycaemic control, the meal increased resting FBF, but did not impair $\triangle \mathrm{FBF}$ or $r \mathrm{FBF}$ ( $p=0.37$ and $p=0.79$ during acetylcholine infusion, $p=0.14$ and $p=0.87$ post ischaemia). In terms of correlation analysis there was a strong inverse correlation between plasma insulin and $\triangle \mathrm{FBF}$ during acetylcholine infusion both in the fasting state $(r=-0.429, p<0.05$ for low-dose acetylcholine and $r=-0.637, p<0.0005$ for high-dose acetylcholine) and in the postprandial state $(r=-0.451, p<0.05$ for low-dose and $r=-0.513, p<0.005$ for high-dose). The postprandial state was associated with a parallel upward shift of the corresponding regression lines along the $y$ axis without a significant change in the slope. Figure 3 illustrates the correlation between $\triangle F B F$ during high-dose acetylcholine infusion and plasma insulin in the fasting and postprandial state, marking participants of the non-diabetic group and the diabetic group separately. Pre- and postprandial $\triangle F B F$ did not correlate with other glycaemic indices, cardiovascular risk markers or lipoproteins.

\section{Discussion}

We demonstrated that in resistance vessels, postprandial endothelium-dependent vasodilation (EDV) does not differ from fasting EDV, despite the exaggerated metabolic excursions in participants with Type 2 diabetes. After 3 months of intensive glycaemic control, we found a reduction in postprandial metabolic excursions, but postprandial EDV only improved to the same degree as fasting EDV.
Fasting endothelial function in Type 2 diabetes. Consistent with our results, many studies have convincingly demonstrated that fasting EDV, both in the microvasculature and the macrovasculature, is impaired in patients with Type 2 diabetes compared with in healthy controls $[11,12]$. A number of causes of endothelial dysfunction in Type 2 diabetes have been proposed, such as increased oxidative stress, uncoupling of nitric oxide synthase and protein kinase $\mathrm{C}$ activation [13]. Many [14, 15, 16], but not all [17, 18] studies report improvement in endothelial function with optimised glucose regulation through intensified insulin or metformin therapy, which is consistent with our findings.

Postprandial endothelial function in the macrovasculature. The effect of a physiological meal on endothelial function in the macrovasculature has been studied extensively $[19,20,21,22,23,24,25,26,27,28,29$, $30,31,32,33]$. Several reports indicate the absence of meal-induced endothelial dysfunction [19, 21, 24, 30], but the majority of studies report an acute impairment of endothelial function in the postprandial state [20, $22,25,26,28,29,31,32,33,34]$, including three studies examining patients with Type 2 diabetes [20, 23, 27]. However, these results have to be interpreted with caution. Flow-mediated dilation (FMD), the surrogate for endothelial function in the macrovasculature, is defined as the ratio of the post-ischaemic vessel diameter to the resting vessel diameter [35]. In the postprandial state, the resting diameter increases independently of endothelial function [19, 21, 23, 25, 26, 27, 29, 30]. Consequently, FMD decreases spuriously and should not be expressed as a ratio [35]. In studies concluding that postprandial FMD reductions 
represent endothelial dysfunction, this bias has not been addressed. Gokce et al. concluded that a high-fat meal does not impair endothelial function, despite significant FMD reductions in their study, as the reductions could be explained by the increase in resting vessel diameter alone [19]. A sex difference in macrovascular endothelial function has been demonstrated, where men showed acute postprandial endothelial dysfunction, but premenopausal women did not [36]. We studied postmenopausal women in order to eliminate the effect of changing oestradiol concentrations on endothelial function.

Postprandial endothelial function in the microvasculature. There are few studies measuring the effect of a physiological meal on EDV in resistance vessels of healthy individuals [20, 21, 37, 38, 39, 40] compared with the wealth of publications examining the macrovasculature. In three of these studies, endothelium-specific vasodilation was measured during intraarterial infusion of endothelial-function-modifying substances (invasive plethysmography) as in our study: Gudmundsson et al. and Muntwyler et al. concluded that there is no impairment of EDV in the postprandial state, which confirms our results [37, 38]. Only Sarabi et al. reported a transient FBF impairment during methacholine infusion 1 hour after a meal containing $34 \%$ fat in ten volunteers [39]. The transient impairment was significant $(p<0.01)$ at $1.7 \mathrm{ml} \cdot \mathrm{min}^{-1} \cdot \mathrm{dl}^{-1}$ but has to be seen in the context of FBF measurements ranging from 13.7 to $20.4 \mathrm{ml} \cdot \mathrm{min}^{-1} \cdot \mathrm{dl}^{-1}$, with SDs of up to $9.5 \mathrm{ml} \cdot \mathrm{min}^{-1} \cdot \mathrm{dl}^{-1}$. Two hours after the meal, vasodilation recovered despite persisting metabolic excursions. When six matched volunteers underwent an identical protocol after a fat-free meal, no transient impairment could be found, suggesting that the fat content and not the postprandial state itself impairs EDV. Given the convincing evidence that nutrient delivery to a tissue bed is closely linked to EDV in the microvasculature [41, 42], it is surprising that in Type 2 diabetes the impact of a physiological meal on endothelial function has not been studied invasively. Noninvasive endothelial function assessment in the microvasculature, i.e. the measurement of post-ischaemic resistance vessel dilation, lacks specificity for endothelial function [43]. In a pilot study, our group found no impairment of the post-ischaemic response 3 hours into the postprandial state [44]. Francesconi et al. described a postprandial reduction in FBF 10 seconds post ischaemia in the diabetes group, but not in the control group [45], measuring maximal vasodilation 10 seconds post ischaemia and not immediately post ischaemia. In our study, the immediate post-ischaemic response did not differ between the postprandial and the fasting state, neither in the diabetic nor in the nondiabetic group. We expanded on the available evidence in Type 2 diabetes by assessing postprandial endothelial function invasively, i.e. during intra-arteri- al infusion of acetylcholine. We found no meal-induced impairment of endothelium-dependent resistance vessel function in either the control or the diabetes group, irrespective of glycaemic control. This finding suggests that the postprandial increases in plasma glucose and lipoproteins do not acutely impair EDV. Consequently exaggerated metabolic excursions found in Type 2 diabetes are unlikely to contribute to insulin resistance via endothelial-vasodilation-impairing mechanisms.

Insulin has vasodilating properties, which are endothelium dependent and mediated by nitric oxide [46]. This effect explains the postprandial increase in resting FBF in our study and all comparable studies cited above. However, we found a strong, inverse correlation of plasma insulin concentration with EDV in the fasting and the postprandial state. Chronic hyperinsulinaemia is believed to affect endothelial function adversely through activation of the mitogen-activated protein kinase pathway [47] and by disturbing the balance between vasoconstricting and vasodilating endothelial mediators [48]. Endothelial dysfunction has been shown to be associated with insulin resistance and the evidence for a causal relationship is mounting [48, 49]. In our study, plasma insulin levels did not reflect the degree of insulin resistance entirely, as most participants injected long-acting insulin 12 hours prior to blood sampling and a fraction of the measured plasma insulin was therefore exogenous. Our findings suggest furthermore that, in contrast to prevailing hyperinsulinaemia, the postprandial insulin surge does not disturb endothelial function. The meal-induced increase in insulin was not associated with impaired EDV and did not alter correlations between plasma insulin and EDV.

Other findings. The marked difference in expiration : inspiration ratio implies a profound, functional impairment of the parasympathetic, autonomous nervous system among participants in the diabetic group. A major role of the parasympathetic nervous system in meal-induced blood flow changes is therefore unlikely, as these responses did not differ between the nondiabetic and the diabetic group. Its contribution to the pre-existing impairment of vasodilation in the diabetic group, however, is conceivable. We were unable to demonstrate a consistent correlation of $\mathrm{HbA}_{1} \mathrm{c}$, plasma glucose, lipoprotein fractions and cardiovascular risk markers with EDV. Currently available data on such correlations are contradictory, possibly reflecting the need for a larger sample size.

Study limitations. We infused the nitric oxide donor acetylcholine only, as the nitric oxide dependence of impaired vasodilation in Type 2 diabetes has been established. However, it is conceivable that there is an impairment of non-nitric-oxide-dependent vasodilation in the postprandial state. In order to minimise ar- 
terial line complications and to not miss the meal response in the non-diabetic group we measured EDV 3 hours after the meal, the time point at which the postprandial triglycerides would have increased to the greatest extent but would not have peaked in the diabetic group [23, 27, 40]. Data from studies measuring EDV once in 3 hours, or serially after a meal challenge suggests that endothelial impairment occurs within 3 hours in healthy volunteers $[25,26,28,39$, 40] and patients with Type 2 diabetes [27, 29] at similar or lower postprandial triglyceride elevations than in our study. It is therefore unlikely that we missed the effect of postprandial lipid excursions on endothelial function in the diabetic group due to the timing of EDV measurements. Plethysmography studies were not repeated in the non-diabetic group; we therefore cannot rule out a time effect on endothelial function in the diabetic group. We did not measure blood pressure intra-arterially, limiting accurate vascular resistance calculations. However, this is unlikely to affect results since endothelium-dependent FBF changes were expressed relative to the non-infused arm, assuming blood pressure was similar in both arms.

Conclusions. The ingestion of a physiological, highfat meal is not associated with acute endothelial dysfunction in forearm resistance vessels of non-diabetic and diabetic women, despite exaggerated metabolic excursions among women with diabetes. After 3 months of intensive glycaemic control, improvements in endothelial function did not differ between the fasting and postprandial state, strengthening the notion that postprandial metabolic excursions are not significant modulators of endothelial function.

Acknowledgements. This work was funded by the 1999 Lilly Asia Pacific Women's Health Research and Education Grant. We thank S. Bennett for the development of blood flow analysis software, Christchurch Hospital Pharmacy staff for the preparation of intra-arterial infusions and all volunteers for their selfless support.

\section{References}

1. Reaven G (2002) Metabolic syndrome: pathophysiology and implications for management of cardiovascular disease. Circulation 106:286-288

2. Rodriguez BL, Lau N, Burchfiel CM et al. (1999) Glucose intolerance and 23-year risk of coronary heart disease and total mortality: the Honolulu Heart Program. Diabetes Care 22:1262-1265

3. No authors listed (1999) Glucose tolerance and mortality: comparison of WHO and American Diabetes Association diagnostic criteria. The DECODE study group. European Diabetes Epidemiology Group. Diabetes Epidemiology: Collaborative Analysis of Diagnostic Criteria in Europe [see comments]. Lancet 354:617-621

4. Parks EJ (2001) Recent findings in the study of postprandial lipemia. Curr Atheroscler Rep 3:462-470

5. Kuvin JT, Patel AR, Sliney KA et al. (2001) Peripheral vascular endothelial function testing as a noninvasive indi- cator of coronary artery disease. J Am Coll Cardiol 38:1843-1849

6. Suwaidi JA, Hamasaki S, Higano ST, Nishimura RA, Holmes DR Jr, Lerman A (2000) Long-term follow-up of patients with mild coronary artery disease and endothelial dysfunction. Circulation 101:948-954

7. Lefebvre PJ, Scheen AJ (1998) The postprandial state and risk of cardiovascular disease. Diabet Med 15 [Suppl 4]:S63-S68

8. Raitakari OT, Celermajer DS (2000) Testing for endothelial dysfunction. Ann Med 32:293-304

9. Golay A (2000) Are postprandial triglyceride and insulin abnormalities neglected cardiovascular risk factors in type 2 diabetes? Eur J Clin Invest 30 [Suppl 2]:12-18

10. Benjamin N, Calver A, Collier J, Robinson B, Vallance P, Webb D (1995) Measuring forearm blood flow and interpreting the responses to drugs and mediators. Hypertension 25:918-923

11. Johnstone MT, Creager SJ, Scales KM, Cusco JA, Lee BK, Creager MA (1993) Impaired endothelium-dependent vasodilation in patients with insulin-dependent diabetes mellitus. Circulation 88:2510-2516

12. McVeigh GE, Brennan GM, Johnston GD et al. (1992) Impaired endothelium-dependent and independent vasodilation in patients with type 2 (non-insulin-dependent) diabetes mellitus. Diabetologia 35:771-776

13. Hink U, Li H, Mollnau H et al. (2001) Mechanisms underlying endothelial dysfunction in diabetes mellitus. Circ Res 88:E14-E22

14. Vehkavaara S, Makimattila S, Schlenzka A, Vakkilainen J, Westerbacka J, Yki-Jarvinen H (2000) Insulin therapy improves endothelial function in type 2 diabetes. Arterioscler Thromb Vasc Biol 20:545-550

15. Mather KJ, Verma S, Anderson TJ (2001) Improved endothelial function with metformin in type 2 diabetes mellitus. J Am Coll Cardiol 37:1344-1350

16. Gaenzer H, Neumayr G, Marschang P et al. (2002) Effect of insulin therapy on endothelium-dependent dilation in type 2 diabetes mellitus. Am J Cardiol 89:431-434

17. Yudkin JS, Panahloo A, Stehouwer C et al. (2000) The influence of improved glycaemic control with insulin and sulphonylureas on acute phase and endothelial markers in Type II diabetic subjects. Diabetologia 43:1099-1106

18. Bagg W, Whalley GA, Gamble G, Drury PL, Sharpe N, Braatvedt GD (2001) Effects of improved glycaemic control on endothelial function in patients with type 2 diabetes. Intern Med J 31:322-328

19. Gokce N, Duffy SJ, Hunter LM, Keaney JF, Vita JA (2001) Acute hypertriglyceridemia is associated with peripheral vasodilation and increased basal flow in healthy young adults. Am J Cardiol 88:153-159

20. Anderson RA, Evans ML, Ellis GR et al. (2001) The relationships between post-prandial lipaemia, endothelial function and oxidative stress in healthy individuals and patients with type 2 diabetes. Atherosclerosis 154:475-483

21. Raitakari OT, Lai N, Griffiths K, McCredie R, Sullivan D, Celermajer DS (2000) Enhanced peripheral vasodilation in humans after a fatty meal. J Am Coll Cardiol 36:417-422

22. Evans M, Anderson RA, Graham J et al. (2000) Ciprofibrate therapy improves endothelial function and reduces postprandial lipemia and oxidative stress in type 2 diabetes mellitus. Circulation 101:1773-1779

23. Fard A, Tuck CH, Donis JA et al. (2000) Acute elevations of plasma asymmetric dimethylarginine and impaired endothelial function in response to a high-fat meal in patients with type 2 diabetes. Arterioscler Thromb Vasc Biol 20:2039-2044 
24. Funada J, Sekiya M, Hamada M, Hiwada K (2002) Postprandial elevation of remnant lipoprotein leads to endothelial dysfunction. Circ J 66:127-132

25. Marchesi S, Lupattelli G, Schillaci G et al. (2000) Impaired flow-mediated vasoactivity during post-prandial phase in young healthy men. Atherosclerosis 153:397-402

26. Plotnick GD, Corretti MC, Vogel RA (1997) Effect of antioxidant vitamins on the transient impairment of endothelium-dependent brachial artery vasoactivity following a single high-fat meal [see comments]. JAMA 278:1682-1686

27. Shige H, Ishikawa T, Suzukawa M et al. (1999) Endothelium-dependent flow-mediated vasodilation in the postprandial state in type 2 diabetes mellitus. Am J Cardiol 84:1272-1274

28. Siepi D, Marchesi S, Lupattelli G et al. (2002) Postprandial endothelial impairment and reduced glutathione levels in postmenopausal women. Ann Nutr Metab 46:32-37

29. Vogel RA, Corretti MC, Plotnick GD (1997) Effect of a single high-fat meal on endothelial function in healthy subjects. Am J Cardiol 79:350-354

30. Williams MJ, Sutherland WH, McCormick MP, Yeoman D, de Jong SA, Walker RJ (2001) Normal endothelial function after meals rich in olive or safflower oil previously used for deep frying. Nutr Metab Cardiovasc Dis 11:147-152

31. Wilmink HW, Banga JD, Hijmering M, Erkelens WD, Stroes ES, Rabelink TJ (1999) Effect of angiotensinconverting enzyme inhibition and angiotensin II type 1 receptor antagonism on postprandial endothelial function. J Am Coll Cardiol 34:140-145

32. Wilmink HW, Stroes ES, Erkelens WD et al. (2000) Influence of folic acid on postprandial endothelial dysfunction. Arterioscler Thromb Vasc Biol 20:185-188

33. Wilmink HW, Twickler MB, Banga JD et al. (2001) Effect of statin versus fibrate on postprandial endothelial dysfunction: role of remnant-like particles. Cardiovasc Res 50:577-582

34. Williams MJ, Sutherland WH, McCormick MP, de Jong SA, Walker RJ, Wilkins GT (1999) Impaired endothelial function following a meal rich in used cooking fat. J Am Coll Cardiol 33:1050-1055

35. Corretti MC, Anderson TJ, Benjamin EJ et al. (2002) Guidelines for the ultrasound assessment of endothelial-dependent flow-mediated vasodilation of the brachial artery: a report of the International Brachial Artery Reactivity Task Force. J Am Coll Cardiol 39:257-265

36. Schillaci G, Marchesi S, Siepi D et al. (2001) Gender differences in postprandial endothelial function. Am J Cardiol $87: 1323-1325$
37. Gudmundsson GS, Sinkey CA, Chenard CA, Stumbo PJ, Haynes WG (2000) Resistance vessel endothelial function in healthy humans during transient postprandial hypertriglyceridemia. Am J Cardiol 85:381-385

38. Muntwyler J, Sutsch G, Kim JH et al. (2001) Post-prandial lipaemia and endothelial function among healthy men. Swiss Med Wkly 131:214-218

39. Sarabi M, Fugmann A, Karlstrom B, Berne C, Lithell H, Lind L (2001) An ordinary mixed meal transiently impairs endothelium-dependent vasodilation in healthy subjects. Acta Physiol Scand 172:107-113

40. Schinkovitz A, Dittrich P, Wascher TC (2001) Effects of a high-fat meal on resistance vessel reactivity and on indicators of oxidative stress in healthy volunteers. Clin Physiol 21:404-410

41. Baron AD, Laakso M, Brechtel G, Hoit B, Watt C, Edelman SV (1990) Reduced postprandial skeletal muscle blood flow contributes to glucose intolerance in human obesity. J Clin Endocrinol Metab 70:1525-1533

42. Coggins M, Lindner J, Rattigan S et al. (2001) Physiologic hyperinsulinemia enhances human skeletal muscle perfusion by capillary recruitment. Diabetes 50:2682-2690

43. Engelke KA, Halliwill JR, Proctor DN, Dietz NM, Joyner MJ (1996) Contribution of nitric oxide and prostaglandins to reactive hyperemia in human forearm. J Appl Physiol 81:1807-1814

44. Shand BI, Scott RS, Florkowski CM, Reynolds, Ikram H (2001) A comparison of post-prandial changes in flow-mediated dilatation in patients with type 2 diabetes with and without macroalbuminuria. Diabetes Nutr Metab 14:201-206

45. Francesconi M, Koizar C, Wascher TC (2001) Postprandial impairment of resistance vessel function in insulin treated patients with diabetes mellitus type-2. Clin Physiol 21:300-307

46. Steinberg HO, Brechtel G, Johnson A, Fineberg N, Baron AD (1994) Insulin-mediated skeletal muscle vasodilation is nitric oxide dependent. A novel action of insulin to increase nitric oxide release. J Clin Invest 94:1172-1179

47. Hsueh WA, Law RE (1998) Cardiovascular risk continuum: implications of insulin resistance and diabetes. Am J Med 105:4S-14S

48. Mather K, Anderson TJ, Verma S (2001) Insulin action in the vasculature: physiology and pathophysiology. J Vasc Res 38:415-422

49. Baron AD (1999) Vascular reactivity. Am J Cardiol $84: 25 \mathrm{~J}-27 \mathrm{~J}$ 